Research Paper

\title{
Sorafenib Monotherapy Versus Sorafenib Combined with Regional Therapies for Hepatocellular Carcinoma Patients with Pulmonary Oligometastases: A Propensity Score-matched Analysis
}

\author{
Jinbin Chen ${ }^{1,2^{*}}$, Shixun $\mathrm{Lu}^{1,3^{*}}$,Yaojun Zhang ${ }^{1,2}$, Li Xu ${ }^{1,2}$, Jiancong Chen ${ }^{1,2}$, Juncheng Wang ${ }^{1,2}$, Minshan \\ Chen ${ }^{1,2}$, Rongxin Zhang ${ }^{1,4}{ }^{\bowtie}$, Zhongguo Zhou ${ }^{1,2} \bowtie$ \\ 1. Sun Yat-sen University Cancer Center; State Key Laboratory of Oncology in South China; Collaborative Innovation Center for Cancer Medicine, Guangzhou, \\ Guangdong 510060, P. R. China \\ 2. Department of Hepatobiliary Oncology, Sun Yat-sen University Cancer Center, Guangzhou, Guangdong 510060, P. R. China. \\ 3. Department of Pathology, Sun Yat-sen University Cancer Center, Guangzhou, Guangdong 510060, P. R. China. \\ 4. Department of Colorectal Cancer, Sun Yat-sen University Cancer Center, Guangzhou, Guangdong 510060, P. R. China \\ * Jinbin Chen and Shixun Lu contributed equally to this work.
}

$\square$ Corresponding author: Rongxin Zhang and Zhongguo Zhou, Department of Hepatobiliary Oncology, Sun Yat-sen University Cancer Center, 651 Dongfeng Road East, Guangzhou, Guangdong 510060, P. R. China. Tel: +86-20-87343828; Fax: +86-20-87343585; Email: zhangrx@sysucc.org.cn or zhouzhg@sysucc.org.cn.

(c) Ivyspring International Publisher. This is an open access article distributed under the terms of the Creative Commons Attribution (CC BY-NC) license (https://creativecommons.org/licenses/by-nc/4.0/). See http://ivyspring.com/terms for full terms and conditions.

Received: 2017.12.26; Accepted: 2018.03.15; Published: 2018.04.19

\begin{abstract}
Background: Sorafenib has been recommended as the standard therapy for advanced HCC with extrahepatic metastasis. The purpose of this retrospective study was to investigate the difference in overall survival (OS) between treatments with sorafenib combined with regional therapies versus sorafenib monotherapy in hepatocellular carcinoma ( $\mathrm{HCC})$ patients with pulmonary oligometastases.

Methods: The study retrospectively enrolled 95 consecutive patients who underwent sorafenib therapy. A combined group (CG) of 40 patients received regional therapy in addition to sorafenib, and a monotherapy group (MG) of 55 patients received only sorafenib. OS was the primary endpoint, and time to progression (TTP) was the secondary endpoint. Subgroup analysis was further performed to evaluate the differences between the two groups. A propensity score-matched analysis was performed to overcome the bias.

Results: Median OS was significantly longer in the CG than in the MG (18.37 vs. 7.13 months; $P=0.002)$. Multivariate analysis identified three baseline characteristics that were prognostic indicators of OS: macrovascular invasion, regional therapy, and alpha-fetoprotein. Median TTP was significantly longer in the CG than in the MG (2.93 vs. 2.23 months; $P=0.004)$. Further multivariate analysis showed alpha-fetoprotein, total bilirubin, and regional therapy as prognostic indicators of TTP. After propensity score matching, 34 paired patients constituted each group. Patients in the adjusted CG showed a longer OS and TTP than those in the adjusted MG (OS: 18.37 vs. 7.37 months, $P=0.015$; TTP: 3.12 vs. 2.265 months, $P=0.009$ ). Multivariate analysis showed that combining regional therapies was still a prognostic indicator of OS $(P=0.01)$ and TTP $(P=0.001)$. Conclusions: Sorafenib combined with regional therapies may be associated with prolonged OS and TTP in HCC patients with pulmonary oligometastases compared with sorafenib monotherapy.
\end{abstract}

Key words: Hepatocellular carcinoma, pulmonary oligometastases, sorafenib, regional therapy, propensity score match

\section{Introduction}

Hepatocellular carcinoma (HCC) is the third leading cause of cancer-related deaths globally. About 700,000 cases were diagnosed worldwide in 2012 [1]. Because of the occult nature of liver cancers, approximately $80 \%$ of patients are not candidates for radical resection upon diagnosis. Extrahepatic metastasis is one of the most common causes of death in HCC, and the lung is the most common site of 
extrahepatic metastatic HCC [2]. Sorafenib, an oral multikinase inhibitor, has been recommended as the standard therapy for advanced HCC with extrahepatic metastasis on the basis of two phase 3 randomised trials $[3,4]$. Only about $40 \%$ of patients have improved overall survival (OS) with sorafenib treatment [4]. Sorafenib-related adverse events, such as skin reactions, hypertension, diarrhoea, and others, occur in more than $90 \%$ of cases. When severe adverse events occur, sorafenib should be reduced or discontinued.

Researchers are pursuing other strategies to improve the therapeutic response in HCC. Previous studies showed that some regional therapies, such as transcatheter chemoembolization (TACE), radiofrequency ablation (RFA), radioactive ${ }^{125 I}$ seed implantation, and resection for metastasis, are available for palliative treatment in such cases [5]. Some studies reported a benefit to OS after pulmonary metastasis resection in patients with HCC when the primary tumour was controlled well or the metastasis was limited.

In 1995, Hellman et al. proposed the idea of an oligometastatic state, in which a few metastases from various cancers exist before the malignant cells acquire widespread metastatic potential [6]. The term oligometastases indicates an intermediate state of cancer that lies between localized disease and widespread metastases. As chemotherapeutic and biological agents have considerably improved outcomes in patients with advanced HCC, treatment of pulmonary oligometastases is increasingly being performed.

Presently, it is still controversial whether combining sorafenib with other therapies could prolong OS of patients with pulmonary oligometastases. We therefore performed a study to investigate the effect of sorafenib combined with regional therapies.

\section{Materials and Methods}

\section{Population and study design}

We conducted a retrospective study that enrolled 95 consecutive HCC patients with pulmonary oligometastases who had received sorafenib therapy in the Sun Yat-sen University Cancer Center between June 2013 and June 2016. This study was approved by the Institutional Review Board of Sun Yat-sen University Cancer Center in Guangzhou, China, and conducted in accordance with approved guidelines. The cases were selected according to the following criteria, and all included subjects had signed the informed consent.
Inclusion criteria: (1) Diagnosed with HCC based on the criteria of the European Association for the Study of the Liver[7]. Pathological diagnosis was only required if the clinical diagnosis was not clear. (2) Pulmonary oligometastases (one to five lesions) revealed by chest computed tomography (CT) scan. (3) Eastern Cooperative Oncology Group (ECOG) performance status of 0-2. (4) Child-Pugh class A or B (class B patients with scores no greater than 8). (5) Sorafenib treatment for at least 4 weeks. (6) Concomitant antiviral systemic therapy was allowed. (7) Patients whose disease had progressed after they received local treatment were allowed.

Exclusion criteria: (1) Unclear diagnosis. (2) HCC combined with other cancer. (3) Previously received molecularly targeted therapies or any other systemic treatment. (4) HCC combined with severe cardio-cerebral diseases, renal, or haematological disease. (5) Incomplete clinical data. (6) Life expectancy of less than 12 weeks.

The subjects were divided into two groups: the monotherapy group (MG), who were treated with sorafenib only; and the combined group (CG), who were treated with sorafenib combined with other regional therapies. MG patients received oral sorafenib (Nexavar Bayer Health Care AG, Leverkusen, Germany, $400 \mathrm{mg}$, twice daily) without any other therapy. However, patients in the CG received other therapies, including transcatheter arterial chemoembolization (TACE), radiofrequency ablation (RFA), radioactive 125I seed implantation and pulmonary metastasis resection, while undergoing sorafenib therapy. Sorafenib dose was allowed to reduce when severe complications were occurred.

\section{Statistical analysis}

OS was the primary endpoint of the analysis, defined as the time from initiation of sorafenib therapy to death or to the last follow-up date in patients whose data were censored. Data from patients who died without tumour progression were recorded and a Fine-Gray competing risk analysis was supposed to reduce the interference from competing risk events which death without tumor progression. The secondary endpoint was time to progression (TTP) based on the modified Response Evaluation Criteria in Solid Tumors (mRECIST) on the basis of independent radiologic review. TTP was defined as the period from initiation of sorafenib therapy to first progression diagnosed by CT or magnetic resonance imaging (MRI) scan. Other related items were recorded retrospectively, including age, sex, number and greatest diameter of liver nodules, macrovascular invasion, other extrahepatic metastases, liver function 
parameters, alpha-fetoprotein (AFP), and treatment history before sorafenib therapy.

Statistical analysis was performed with SPSS 22.0 software. A propensity score-matched analysis was performed to overcome the bias from the different distribution in the two groups. A 1:1 match without replacement was performed through the nearest available matching, setting the calliper as 0.2. The following variables were matched for age, gender, ECOG score, tumour size $(>5 \mathrm{~cm}$ or not), macrovascular invasion, number of tumours, other organ metastasis, lymph node metastasis and levels of AFP, albumin and total bilirubin. Student's $t$-test was used to compare continuous variables when the data was distributed normally. Mann-Whitney U test was used to compare skewed data, and the chi-square or Fisher's exact test was used for categorical variables. OS and TTP were estimated by Kaplan-Meier analysis, and the curves were compared by the log-rank test. The Cox proportional hazards model was used for multivariate analysis of survival. All variables found significant in the univariate analysis were included in the multivariate model. Subgroup analysis was further performed to evaluate the difference between monotherapy and combined therapy in selected populations. A two-sided $P$ value $<0.05$ was considered to indicate statistical significance.

\section{Results}

\section{Baseline characteristics}

More than 500 cases of HCC were diagnosed with pulmonary metastases in our cancer centre during the period from June 2013 to June 2016, including initial diagnosis and post-treatment progression. Owing to the limitations of the economic situation and liver function, sorafenib therapy was administered to 180 patients, among which 109 HCC patients with pulmonary oligometastases who had been treated with sorafenib were identified initially. Seventeen patients were excluded on the basis of the exclusion criteria. Among them, three patients had a previous history of cancer, and two had received other systemic treatments. The diagnosis of pulmonary metastases in one case was not clear. The remaining eight excluded patients had received sorafenib therapy for less than 4 weeks. Two patients of these eight patients simultaneously met the exclusion criterion of having a life expectancy of less than 12 weeks. Both of them were diagnosed with multi-organ metastases that progressed explosively before the initiation of sorafenib treatment. Finally, 95 patients were included in our study. Nearly all of the patients were male $(91 / 95,95.79 \%)$, and the median age was 49.49 years (range, 22 to 79 ). The most frequent type of hepatitis was hepatitis B virus (HBV) infection $(83 / 95,87.37 \%)$. Only two patients had hepatitis $\mathrm{C}$ virus $(\mathrm{HCV})$ infection. Most patients had preserved liver function with Child-Pugh status A (87/95, 91.58\%). Most patients were in the ECOG performance status of 0 and 1 (62 and 31, respectively). Only two of them were in the ECOG performance status of 2 . In addition to pulmonary metastases, multiple nodules (52/95, 64.74\%), macrovascular invasion $(35 / 95,36.84 \%)$, and other extrahepatic metastases $(19 / 95,20 \%)$ could be identified. In 25 patients, no tumour could be discovered in the liver, even though pulmonary metastasis was clearly present. The median duration of sorafenib therapy was 156 days (range, 30 to 881 days).

The 95 patients were then assigned to the CG (40 patients) or the MG (55 patients). The baseline demographic and disease characteristics of the patients are shown in Table 1 . There were no significant differences between the $\mathrm{CG}$ and the $\mathrm{MG}$, except for total bilirubin and lymph node metastasis. The average total bilirubin was significantly higher in MG than in CG (19.28 vs. $13.83 \mu \mathrm{mol} / \mathrm{L}, P=0.009)$, and further lymph node metastases were identified in MG than in CG (10/45 in MG and 1/39 in CG, respectively, $P=0.042)$.

\section{Survival analysis of OS}

All the patients who reached the endpoint were resulted from multi-organ failure by tumor progression. Fine-Gray competing risk analysis was not applicable in this group of patients. The median follow-up time was 8.37 months (range, 1.77 to 33.23 months). Median OS was significantly longer in the CG than in the MG (18.37 vs. 7.13 months; HR, 0.416; 95\% CI, $0.236-0.731 ; P=0.002$ ) (Figure 1 ). The survival rate at 1 year was $67.5 \%$ in the CG and $41.8 \%$ in the MG. This significant survival benefit represented a $58.4 \% \%$ relative reduction in the risk of death.

The univariate analysis performed to determine baseline prognostic factors for better survival showed that in addition to regional therapy, lower burden of tumour (small liver nodule, single hepatic nodule, absence of macrovascular invasion), lower AFP level, higher albumin (ALB) level, higher total bilirubin level, and prior history of therapy were statistically significant $(P<0.05)$ (Table 2$)$. A multivariate analysis with the use of a Cox proportional hazards model, which included all the significant factors on univariate analysis, identified three baseline characteristics that were prognostic indicators of OS: macrovascular invasion $(P=0.007 ; \mathrm{HR}, 2.260 ; 95 \% \mathrm{CI}$, 1.256-4.067), regional therapy $(P=0.002$; $\mathrm{HR}, 0.370$; 
95\% CI, 0.199-0.688), and AFP level ( $P=0.005$; HR, $2.458 ; 95 \%$ CI, 1.303-4.636) (Table 2). After adjustment for these prognostic factors, the effect of combining regional therapy on OS remained significant.

Table 1. Baseline demographic and disease characteristics of patients

\begin{tabular}{|c|c|c|c|c|c|c|}
\hline \multirow[t]{2}{*}{ Characteristic } & \multicolumn{3}{|c|}{ Pre-match } & \multicolumn{3}{|c|}{ Post-match } \\
\hline & CG & MG & $P$ Value & CG & MG & $P$ Value \\
\hline No. of cases & 40 & 55 & & 34 & 34 & \\
\hline Sex & & & 0.060 & & & 1.000 \\
\hline M & 36 & 55 & & 34 & 34 & \\
\hline $\mathrm{F}$ & 4 & 0 & & 0 & 0 & \\
\hline Age (years) & $\begin{array}{l}49.83 \pm \\
10.325\end{array}$ & $\begin{array}{l}49.25 \pm \\
11.447\end{array}$ & 0.803 & $\begin{array}{l}50.65 \pm \\
11.026\end{array}$ & $\begin{array}{l}51.09 \pm \\
10.223\end{array}$ & 0.548 \\
\hline $\operatorname{HBV}(+/-)$ & $37 / 3$ & $46 / 9$ & 0.199 & $32 / 2$ & $28 / 6$ & 0.259 \\
\hline $\mathrm{HCV}(+/-)$ & $1 / 39$ & $1 / 54$ & 1.000 & $1 / 33$ & $1 / 33$ & 1.000 \\
\hline ECOG & & & 0.882 & & & 0.592 \\
\hline 0 & 27 & 35 & & 23 & 19 & \\
\hline 1 & 12 & 19 & & 20 & 14 & \\
\hline 2 & 1 & 1 & & 1 & 1 & \\
\hline Prior treatment & & & 0.056 & & & 0.259 \\
\hline Y & 38 & 45 & & 32 & 28 & \\
\hline $\mathrm{N}$ & 2 & 10 & & 2 & 6 & \\
\hline Child-Pugh class & & & 0.516 & & & 0.673 \\
\hline A & 38 & 49 & & 32 & 30 & \\
\hline B & 2 & 6 & & 2 & 4 & \\
\hline Tumours in liver & & & 0.487 & & & 0.431 \\
\hline Y & 28 & 42 & & 22 & 25 & \\
\hline $\mathrm{N}$ & 12 & 13 & & 12 & 9 & \\
\hline No. of tumours & & & 0.932 & & & 0.773 \\
\hline 0 & 12 & 15 & & 10 & 12 & \\
\hline 1 & 7 & 9 & & 7 & 5 & \\
\hline Multiple & 21 & 31 & & 17 & 17 & \\
\hline Size of tumours $(\mathrm{cm})$ & & & 0.852 & & & 0.637 \\
\hline 0 & 12 & 14 & & 12 & 9 & \\
\hline$\leq 5$ & 12 & 19 & & 8 & 11 & \\
\hline$>5$ & 16 & 22 & & 14 & 14 & \\
\hline $\begin{array}{l}\text { Macrovascular } \\
\text { invasion }\end{array}$ & & & 0.238 & & & 0.442 \\
\hline Y & 28 & 32 & & 24 & 21 & \\
\hline $\mathrm{N}$ & 12 & 23 & & 10 & 13 & \\
\hline $\begin{array}{l}\text { Other organ } \\
\text { metastasis }\end{array}$ & & & 1.000 & & & 1.000 \\
\hline Y & 8 & 11 & & 6 & 6 & \\
\hline $\mathrm{N}$ & 32 & 44 & & 28 & 28 & \\
\hline $\begin{array}{l}\text { Lymph node } \\
\text { metastasis }\end{array}$ & & & 0.042 & & & 0.614 \\
\hline Y & 1 & 10 & & 1 & 3 & \\
\hline $\mathrm{N}$ & 39 & 45 & & 33 & 31 & \\
\hline $\operatorname{AFP}(\mathrm{ng} / \mathrm{ml})$ & & & 0.775 & & & 1.000 \\
\hline$<400$ & 17 & 25 & & 15 & 15 & \\
\hline$\geq 400$ & 23 & 30 & & 19 & 19 & \\
\hline $\begin{array}{l}\text { White blood cells } \\
\left(\times 10^{9} / \mathrm{L}\right)\end{array}$ & $\begin{array}{l}6.58 \pm \\
2.35\end{array}$ & $\begin{array}{l}6.95 \pm \\
2.62\end{array}$ & 0.499 & $\begin{array}{l}6.70 \pm \\
2.47\end{array}$ & $\begin{array}{l}7.04 \pm \\
2.87\end{array}$ & 0.392 \\
\hline $\begin{array}{l}\text { Platelet count } \\
\left(\times 10^{9} / \mathrm{L}\right)\end{array}$ & $\begin{array}{l}178.08 \pm \\
83.03\end{array}$ & $\begin{array}{l}174.87 \pm \\
98.68\end{array}$ & 0.873 & $\begin{array}{l}173.17 \pm \\
85.94\end{array}$ & $\begin{array}{l}161.96 \pm \\
82.76\end{array}$ & 0.465 \\
\hline Hemoglobin (g/L) & $\begin{array}{l}139.84 \pm \\
20.12\end{array}$ & $\begin{array}{l}141.29 \pm \\
19.74\end{array}$ & 0.738 & $\begin{array}{l}142.96 \pm \\
18.77\end{array}$ & $\begin{array}{l}142.17 \pm \\
22.07\end{array}$ & 0.565 \\
\hline Serum ALT (U/L) & $\begin{array}{l}60.14 \pm \\
43.52\end{array}$ & $\begin{array}{l}51.16 \pm \\
71.95\end{array}$ & 0.496 & $\begin{array}{l}63.61 \pm \\
45.26\end{array}$ & $\begin{array}{l}54.15 \pm \\
84.57\end{array}$ & 0.979 \\
\hline Serum AST (U/L) & $\begin{array}{l}76.12 \pm \\
62.31\end{array}$ & $\begin{array}{l}62.14 \pm \\
43.46\end{array}$ & 0.238 & $\begin{array}{l}80.53 \pm \\
65.12\end{array}$ & $\begin{array}{l}62.84 \pm \\
45.89\end{array}$ & 0.053 \\
\hline $\begin{array}{l}\text { Total bilirubin } \\
(\mu \mathrm{mol} / \mathrm{L})\end{array}$ & $\begin{array}{l}13.83 \pm \\
7.46\end{array}$ & $\begin{array}{l}19.28 \pm \\
10.85\end{array}$ & 0.009 & $\begin{array}{l}14.02 \pm \\
7.77\end{array}$ & $\begin{array}{l}17.67 \pm \\
9.20\end{array}$ & 0.624 \\
\hline $\operatorname{ALB}(\mathrm{g} / \mathrm{L})$ & $\begin{array}{l}40.09 \pm \\
4.97\end{array}$ & $\begin{array}{l}39.95 \pm \\
5.25\end{array}$ & 0.901 & $\begin{array}{l}40.44 \pm \\
4.87\end{array}$ & $\begin{array}{l}40.11 \pm \\
5.61\end{array}$ & 0.155 \\
\hline Prothrombin time (s) & $\begin{array}{l}11.89 \pm \\
1.10\end{array}$ & $\begin{array}{l}12.35 \pm \\
1.14\end{array}$ & 0.064 & $\begin{array}{l}11.94 \pm \\
1.11\end{array}$ & $\begin{array}{l}12.15 \pm \\
1.03\end{array}$ & 0.758 \\
\hline
\end{tabular}

AFP, alpha-fetoprotein; ALB, albumin; ALT, alanine transaminase; AST, aspartate transaminase; $C G$, combined group; $\mathrm{HBV}$, hepatitis $B$ virus; $\mathrm{HCV}$, hepatitis $\mathrm{C}$ virus; MG, monotherapy group.

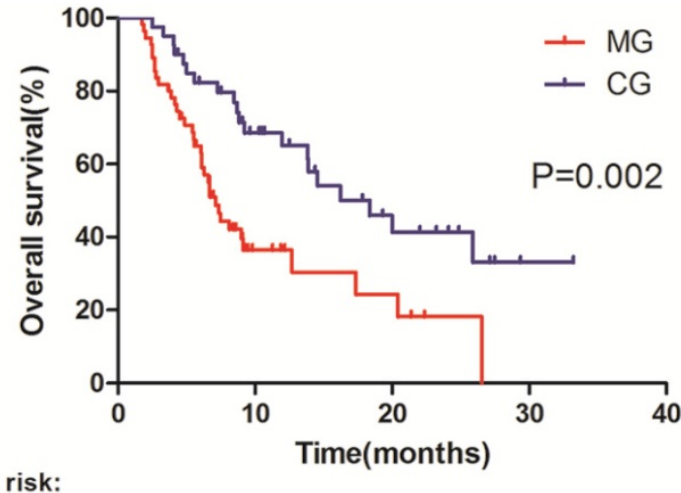

Number at risk:

$\begin{array}{lrrrrr}\text { MG } & 55 & 9 & 4 & 0 & 0 \\ \text { CG } & 40 & 23 & 10 & 1 & 0\end{array}$

Figure 1. Kaplan-Meier analysis of overall survival (OS) in all patients. Data were stratified according to treatment with sorafenib monotherapy (median OS, 18.37 months) and sorafenib combined with regional treatment (median OS, 7.13 months). MG, monotherapy group; CG, combined group.

We performed stratified subgroup survival analyses according to the independent prognostic factors for OS identified in the multivariate analysis. The results of the subgroup analysis were similar to those calculated previously (Figure 2). In the subgroup with macrovascular invasion, median OS was significantly longer in the CG than in the MG (13.8 vs. 4.9 months; $P=0.024$ ) (Figure $2 a$ ). In the subgroup without macrovascular invasion, the difference in OS was also significant (25.9 months in the CG vs. 12.70 months in the MG; $P=0.045$ ) (Figure $2 b$ ). In the subgroup with AFP $<400 \mathrm{ng} / \mathrm{ml}$ (subgroup of low AFP), OS was significantly longer in the CG than in the MG (unreached vs. 9.1 months; $P=0.003$ ) (Figure 2c). The result was similar in the subgroup with AFP $\geq 400 \mathrm{ng} / \mathrm{ml}$ (subgroup of high AFP, 13.8 months in the CG vs. 6.1 months in the MG; $P=0.03$ ) (Figure 2d).

\section{Survival analysis of TTP}

CG patients had a significantly longer median TTP (2.93 months) than did MG patients (2.23 months) (HR, 0.54; 95\% CI, 0.351-0.832; $P=0.004$ ) (Figure 3). On univariate analysis, history of prior therapy, tumour size, number of tumours, macrovascular invasion, lymph node metastases, AFP level, total bilirubin level, and combined regional therapy were associated with TTP. Further multivariate analysis identified AFP level (HR, 2.657; 95\% CI, 1.618-4.361; $P$ $<0.001$ ), combined regional therapy (HR, 0.461; $95 \%$ CI, 0.291-0.730; $P=0.001$ ), and total bilirubin level (HR, 3.670; 95\% CI, 1.603-8.405; $P=0.002$ ) as prognostic indicators of TTP (Table 2 ). 


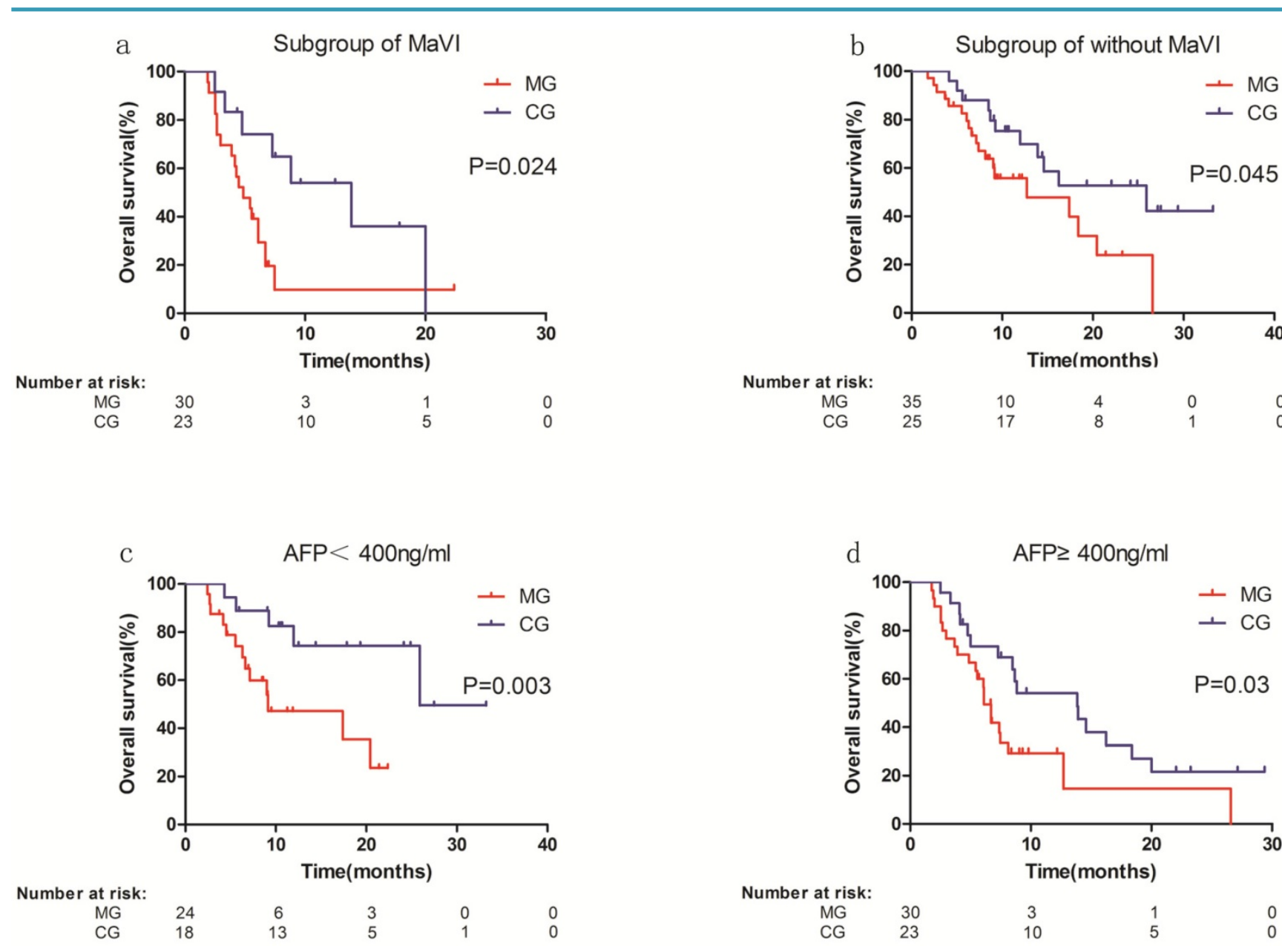

Figure 2. Subgroup analysis of overall survival (OS). a Subgroup with macrovascular invasion (median OS in MG vs. CG: 4.9 vs. 13.8 months). b. Subgroup without macrovascular invasion (median OS in MG vs. CG: 12.70 vs. 25.9 months). c. Subgroup with alpha-fetoprotein (AFP) < $400 \mathrm{ng} / \mathrm{ml}$ (median OS in MG vs. CG: 9.1 vs. unreached). d. Subgroup with AFP $\geq 400 \mathrm{ng} / \mathrm{ml}$ (median OS in MG vs. CG: 6.1 vs. 13.8 months). MG, monotherapy group; CG, combined group; MaVl, macrovascular invasion.

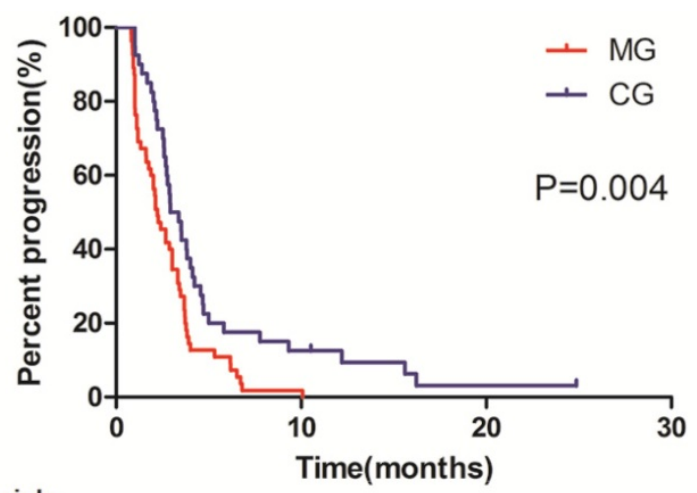

Number at risk:

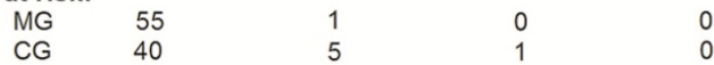

Figure 3. Kaplan-Meier analysis of time to progression (TTP) in all patients. Data were stratified according to treatment with sorafenib monotherapy (median TTP, 2.23 months) and sorafenib combined with regional treatment (median TTP, 2.93 months). MG, monotherapy group; CG, combined group.

\section{Matched analysis}

To confirm the survival benefit of combining regional therapies, the propensity scores were calculated in both groups. We successfully matched 34 patients in the MG and 34 patients in the CG based on their propensity scores. As shown in Table 1, baseline demographic and disease characteristics did not differ between the two matched groups, including total bilirubin and lymph node metastasis, which were significantly different between the two groups before matched analysis. Similarly, the median OS was longer in the matched CG than in the matched MG (18.37 vs. 7.37 months, HR, 0.43; 95\% CI, 0.216-0.849; $P=0.015$ ) (Figure 4a). TTP was still significantly longer in the CG after matched analysis (3.12 months vs. 2.265 months in MG, HR, 0.50; 95\% CI, 0.296-0.842, $P=0.009$ ) (Figure 4b). In multivariable analysis adjusted for other confounders, regional therapies were still determined to be a prognostic indicator of OS (HR, 0.374; 95\% CI, 0.178-0.788; $P=$ 0.01 ) and TTP (HR, 0.384; 95\% CI, (0.218-0.677); $P=$ 0.001 ), and the level of total bilirubin was no longer an independent predictor of OS and TTP after adjustment for the covariates (Table 3). 
Table 2. Univariate and multivariate analysis of variables affecting OS and TTP before propensity score match

\begin{tabular}{|c|c|c|c|c|c|c|}
\hline \multirow[t]{3}{*}{ Variable } & \multicolumn{3}{|l|}{ OS } & \multicolumn{3}{|l|}{ TTP } \\
\hline & Univariate & Multivariate & & Univariate & Multivariate & \\
\hline & $P$ value & Adjusted HR (95\% CI) & $P$ value & $P$ value & Adjusted HR (95\% CI) & $P$ value \\
\hline Prior history of treatment (vs. no) & $<0.001$ & & & $<0.001$ & & \\
\hline Tumour size $\geq 5 \mathrm{~cm}($ vs. $<5 \mathrm{~cm})$ & 0.005 & & & 0.008 & & \\
\hline Multiple tumours (vs. single tumour) & 0.01 & & & 0.01 & & \\
\hline Macrovascular invasion (vs. no) & $<0.001$ & $2.260(1.256-4.067)$ & 0.007 & 0.002 & & \\
\hline Albumin $<35 \mathrm{~g} / \mathrm{L}$ (vs. $\geq 35 \mathrm{~g} / \mathrm{L}$ ) & 0.042 & & & 0.059 & & \\
\hline $\mathrm{AFP} \geq 400 \mathrm{ng} / \mathrm{ml}(\mathrm{vs} .<400 \mathrm{ng} / \mathrm{ml})$ & 0.007 & $2.458(1.303-4.636)$ & 0.005 & 0.003 & $2.657(1.618-4.361)$ & $<0.001$ \\
\hline Total bilirubin $\geq 34 \mu \mathrm{mol} / \mathrm{L}(\mathrm{vs} .<34 \mu \mathrm{mol} / \mathrm{L})$ & 0.01 & & & 0.013 & $3.670(1.603-8.405)$ & 0.002 \\
\hline Combined therapy (vs. monotherapy) & 0.002 & $0.370(0.199-0.688)$ & 0.002 & 0.005 & $0.461(0.291-0.730)$ & 0.001 \\
\hline
\end{tabular}

AFP, alpha-fetoprotein; CI, confidence interval; HR, hazard ratio; OS, overall survival;TTP, time to progression.

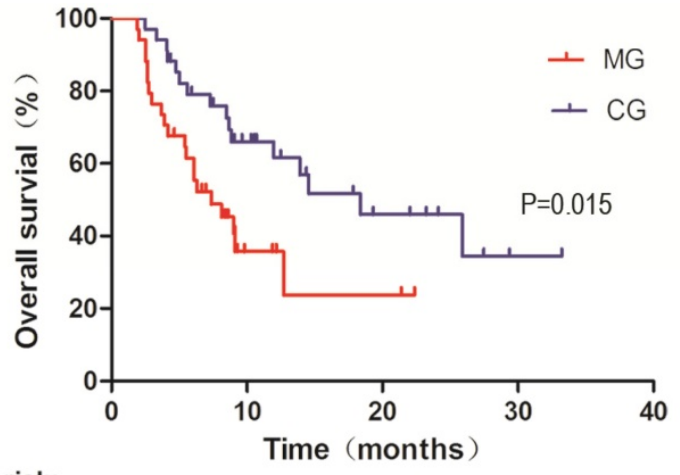

Number at risk:

$\begin{array}{ll}\text { MG } & 34 \\ \text { CG } & 34\end{array}$
6

19
2

8
0

1

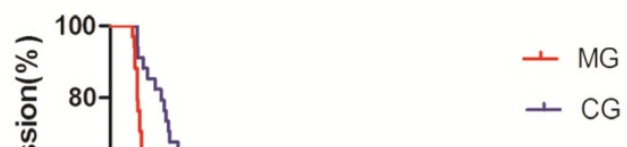

$P=0.009$

Number at risk:

MG $\quad 34$

Figure 4. Kaplan-Meier analysis of overall survival (OS) and time to progression (TTP) in all patients after propensity score match. a. OS data were stratified according to treatment with sorafenib monotherapy (median OS, 18.37 months) and sorafenib combined with regional treatment (median OS, 7.37 months); b. TTP data were stratified according to treatment with sorafenib monotherapy (median TTP, 3.12 months) and sorafenib combined with regional treatment (median TTP, 2.265 months). MG, monotherapy group; CG, combined group.

Table 3. Univariate and multivariate analysis of variables affecting OS and TTP after propensity score match

\begin{tabular}{|c|c|c|c|c|c|c|}
\hline \multirow[t]{3}{*}{ Variable } & \multicolumn{3}{|l|}{ OS } & \multicolumn{3}{|l|}{ TTP } \\
\hline & Univariate & Multivariate & & Univariate & Multivariate & \\
\hline & $P$ value & Adjusted HR (95\% CI) & $P$ value & $P$ value & Adjusted HR (95\% CI) & $P$ value \\
\hline Prior history of treatment (vs. no) & 0.011 & & & 0.02 & & \\
\hline Tumour size $\geq 5 \mathrm{~cm}$ (vs. $<5 \mathrm{~cm})$ & 0.016 & & & 0.028 & & \\
\hline Multiple tumours (vs. single tumour) & 0.034 & & & 0.048 & & \\
\hline Macrovascular invasion (vs. no) & 0.001 & $3.030(1.256-7.299)$ & 0.014 & 0.001 & & \\
\hline Albumin<35 g/L (vs. $\geq 35 \mathrm{~g} / \mathrm{L})$ & 0.02 & & & 0.049 & & \\
\hline $\mathrm{AFP} \geq 400 \mathrm{ng} / \mathrm{ml}(\mathrm{vs} .<400 \mathrm{ng} / \mathrm{ml})$ & 0.002 & $2.924(1.263-6.757)$ & 0.012 & $<0.001$ & $2.114(1.117-4.016)$ & 0.022 \\
\hline Total bilirubin $\geq 34 \mu \mathrm{mol} / \mathrm{L}(\mathrm{vs} .<34 \mu \mathrm{mol} / \mathrm{L})$ & 0.104 & & & 0.283 & & \\
\hline Combined therapy (vs. monotherapy) & 0.018 & $0.374(0.178-0.788)$ & 0.01 & 0.011 & $0.384(0.218-0.677)$ & 0.001 \\
\hline
\end{tabular}

AFP, alpha-fetoprotein; CI, confidence interval; HR, hazard ratio; OS, overall survival; TTP, time to progression.

\section{Discussion}

We conclude from this retrospective study that combining sorafenib therapy with regional therapies may be associated with prolonged OS and TTP in HCC patients with pulmonary oligometastases, in both the initial groups and the propensity score-matched patient groups. The benefits of the combined treatments were confirmed through multivariate analysis after adjustment for other predictors. Stratification according to macrovascular invasion and AFP level demonstrated the benefits of combined therapy in all subgroups.
Oligometastases is a special state of cancer that lies between localized disease and widespread metastases in the process of tumour spread. Limited pulmonary lesions may be curable in that situation. Rapid progress in such technologies as CT, MRI, and positron emission tomography computed tomography and their integration makes it easier to detect a state of oligometastases, including patients with isolated pulmonary metastases. The lung is the most common site of extrahepatic metastases [8]. Extrahepatic metastasis is one of the most important predictors of poor OS $[9,10]$. Several studies have investigated therapies for oligometastases, with 
promising results [11-13]. Niibe et al. reported a 2-year OS rate of $65.7 \%$ in patients with pulmonary oligometastases [12]. When the liver tumours were relatively limited, without macrovascular invasion or lower AFP level, OS in both groups was better.

Regional therapy may help control the disease on the background of sorafenib treatment. In our study, the TTP and OS were longer in the CG. However, the improvement in the TTP was only 0.7 month (2.93 vs. 2.23 months), whereas the improvement in the OS was much longer (18.37 vs. 7.13 months). The post-progression treatments may contribute to the difference in the OS. In some cases, sorafenib treatment was stopped after progression, and regional therapy could potentially play an important role in prolonging the OS. However, further investigations should be conducted in a larger population to determine how the post-progression treatment affects the OS in such patients.

Potential benefits of combining sorafenib with other regional therapies have been reported in a number of previous studies [5, 14-17]. However, the effect of regional therapies in patients with pulmonary oligometastases is still controversial. Some researchers concluded that the therapeutic effect of sorafenib may be similar whether or not extrahepatic metastases exist [18]. Intrahepatic tumour stage can also be a significant and independent prognostic factor. Among patients with extrahepatic metastases, those with intrahepatic tumours at an earlier stage had a better prognosis than patients with more advanced tumours [19].

Hepatic therapies could possibly improve the therapeutic effect of sorafenib by controlling the progression of intrahepatic tumours[20]. Most patients with advanced HCC die of liver failure due to intrahepatic tumour progression rather than from progression of extrahepatic metastatic disease. TACE has been chosen as a palliative treatment. It has been demonstrated that local therapies, including TACE, can induce overproduction of vascular endothelial growth factor, which may facilitate disease progression and metastasis [21, 22]. Patients whose intrahepatic tumours had a complete or partial response after treatment with TACE had significantly longer OS than those who did not have an objective tumour response (521 vs. 170 days) [23]. Several studies have shown that sorafenib combined with TACE may be more effective than sorafenib alone in unresectable advanced HCC, with median OS ranging from 7.5 months to as long as 27.0 months $[5,15,24$, 25]. In our study, TACE was the most common regional therapy; 27 patients had received TACE treatment, and nine had received two or more sessions. Besides TACE, other regional therapies could be considered. Regional ablation has been demonstrated to be a safe modality for local control of pulmonary tumours. As a curative therapy, regional ablation maybe a potential option in advanced cases[26]. Hiraki et al. reported that RFA of 83 pulmonary metastases resulting from HCC was effective and safe in selected patients, with an effectiveness rate of $92 \%$ and a significant improvement in survival rate [27]. Another study suggested that imaging-guided percutaneous ablation techniques should be considered as a useful option for the treatment of extrahepatic disease, including pulmonary metastases, in patients with HCC [28]. Similar results were found in studies of 125I implantation, with a median OS of 14.5 to 21 months $[29,30]$. Regrettably, studies of such regional therapies for lung metastases in combination with sorafenib are rare. Li et al. reported the results of $125 \mathrm{I}$ seed brachytherapy combined with sorafenib in the treatment of patients with multiple lung metastases after orthotopic liver transplantation. The results were promising, but only eight patients were enrolled in the study [29].

Another choice for treatment of pulmonary metastases is pulmonary therapy. When the metastasis is limited and is amendable to resection, pulmonary metastasectomy (PMT) in patients with recurrent HCC confined to the lung can enhance disease control and prolong survival [31]. Whether combining PMT and sorafenib treatment could prolong survival further in comparison with sorafenib monotherapy remains unclear. The other choice for pulmonary therapy is stereotactic body radiotherapy, which has been reported effective and safe in pulmonary oligometastases [11,32].

There are several limitations to our study. First, because this was a retrospective study, though confirmed by propensity score matching analysis, the choice of therapy was influenced by the individual clinician's experience, and subjective factors could result in bias. Second, the numbers of cases for some of the regional therapies, such as PMT and ${ }^{125 I}$ implantation, were not sufficient. The limited numbers of cases would impede further analysis of differences in benefits among these therapies. Further studies with larger numbers of patients are required to determine the effect of regional therapies in patients with HCC receiving sorafenib treatment.

\section{Conclusion}

In conclusion, in a retrospective study comparing sorafenib monotherapy with sorafenib combined with regional therapy for prolonging OS in patients with pulmonary oligometastases, the results indicate that the combined treatment may be 
preferred. We are expecting further prospective randomized studies to test the potential benefit of sorafenib combined with regional therapy in comparison with monotherapy with sorafenib, the current standard of care for HCC with pulmonary oligometastases.

\section{Abbreviations}

AFP: alpha-fetoprotein;

ALB: albumin;

ALT: alanine transaminase;

AST: aspartate transaminase;

CG: combined group;

CI: confidence interval;

CT: computed tomography;

HBV: hepatitis B virus;

HCC: hepatocellular carcinoma;

$\mathrm{HCV}$ : hepatitis $\mathrm{C}$ virus;

HR: hazard ratio;

MG: monotherapy group;

MRI: magnetic resonance imaging;

OS: overall survival;

PMT: pulmonary metastasectomy;

RFA: radiofrequency ablation;

TACE: transcatheter chemoembolization;

TTP: time to progression.

\section{Acknowledgements}

This work was supported by the National Natural Science Foundation of China (No. 81602143) and Sun Yat-sen University Cancer Center physician scientist funding (No. 16zxqk04).

\section{Authors' contributions}

Chen JB and Zhou ZG designed the research and wrote the manuscript. Lu SX, Zhang YJ, Xu L, Chen JC participated in the recruitment of patients and data collection. Wang JC and Zhang RX analysed the data. Chen MS analysed the data, reviewed and edited the manuscript.

\section{Competing Interests}

The authors have declared that no competing interest exists.

\section{References}

1. Torre LA, Bray F, Siegel RL, Ferlay J, Lortet-Tieulent J, Jemal A. Global cancer statistics, 2012. CA Cancer J Clin. 2015; 65: 87-108.

2. Katyal S, Oliver JH, 3rd, Peterson MS, Ferris JV, Carr BS, Baron RL. Extrahepatic metastases of hepatocellular carcinoma. Radiology. 2000; 216: 698-703.

3. Cheng AL, Kang YK, Chen Z, Tsao CJ, Qin S, Kim JS, et al. Efficacy and safety of sorafenib in patients in the Asia-Pacific region with advanced hepatocellular carcinoma: a phase III randomised, double-blind, placebo-controlled trial. Lancet Oncol. 2009; 10: 25-34.

4. Llovet JM, Ricci S, Mazzaferro V, Hilgard P, Gane E, Blanc JF, et al. Sorafenib in advanced hepatocellular carcinoma. N Engl J Med. 2008; 359: 378-90.

5. Bai W, Wang YJ, Zhao Y, Qi XS, Yin ZX, He CY, et al. Sorafenib in combination with transarterial chemoembolization improves the survival of patients with unresectable hepatocellular carcinoma: A propensity score matching study. Journal of Digestive Diseases. 2013; 14: 181-90.

6. Hellman S, Weichselbaum RR. Oligometastases. J Clin Oncol. 1995; 13: 8-10.

7. European Association for The Study of the L, European Organisation For R, Treatment of C. EASL-EORTC clinical practice guidelines: management of hepatocellular carcinoma. Journal of hepatology. 2012; 56: 908-43.

8. Llovet JM, Burroughs A, Bruix J. Hepatocellular carcinoma. Lancet. 2003; 362: $1907-17$.

9. Llovet JM, Bru C, Bruix J. Prognosis of hepatocellular carcinoma: The BCLC staging classification. Seminars in Liver Disease. 1999; 19: 329-38.

10. Poon RTP, Fan ST, Lo CM, Liu CL, Wong J. Intrahepatic recurrence after curative resection of hepatocellular carcinoma - Long-term results of treatment and prognostic factors. Annals of Surgery. 1999; 229: 216-22.

11. Hatime $\mathrm{M}$, Elmorabit $\mathrm{B}$, Elkhoti $\mathrm{Y}$, Touch $\mathrm{S}$, Tanguy $\mathrm{R}$, Mornex $\mathrm{F}$. [Oligometastatic disease, a new concept: stereotactic irradiation for lung metastases. Literature review]. Cancer radiotherapie: journal de la Societe francaise de radiotherapie oncologique. 2012; 16: 351-7.

12. Niibe $Y$, Yamashita H, Sekiguchi K, Takahashi W, Shiraishi K, Okuma K, et al. Stereotactic Body Radiotherapy Results for Pulmonary Oligometastases: A Two-Institution Collaborative Investigation. Anticancer research. 2015; 35: 4903-8.

13. Omae K, Hiraki T, Gobara H, Iguchi T, Fujiwara H, Matsui Y, et al. Long-Term Survival after Radiofrequency Ablation of Lung Oligometastases from Five Types of Primary Lesions: A Retrospective Evaluation. Journal of vascular and interventional radiology: JVIR. 2016; 27: 1362-70.

14. Choi GH, Shim JH, Kim M-J, Ryu M-H, Ryoo B-Y, Kang Y-K, et al. Sorafenib alone versus sorafenib combined with transarterial chemoembolization for advanced-stage hepatocellular carcinoma: results of propensity score analyses. Radiology. 2013; 269: 603-11.

15. Cosgrove DP, Reyes DK, Pawlik TM, Feng AL, Kamel IR, Geschwind JF. Open-Label Single-Arm Phase II Trial of Sorafenib Therapy with Drug-eluting Bead Transarterial Chemoembolization in Patients with Unresectable Hepatocellular Carcinoma: Clinical Results. Radiology. 2015; 277: 594-603.

16. Cha J, Seong I, Lee IJ, Kim JW, Han KH. Feasibility of sorafenib combined with local radiotherapy in advanced hepatocellular carcinoma. Yonsei Med J. 2013; 54: $1178-85$

17. Lee S, Kim BK, Kim SU, Park Y, Chang S, Park JY, et al. Efficacy of sorafenib monotherapy versus sorafenib-based loco-regional treatments in advanced hepatocellular carcinoma. PloS one. 2013; 8: e77240.

18. Nakano M, Tanaka M, Kuromatsu R, Nagamatsu H, Tajiri N, Satani M, et al. Sorafenib for the treatment of advanced hepatocellular carcinoma with extrahepatic metastasis: a prospective multicenter cohort study. Cancer Med. 2015; 4: 1836-43.

19. Kawaoka T, Aikata H, Kan H, Fujino H, Fukuhara T, Kobayashi T, et al. Clinical outcome and prognostic factors of patients with hepatocellular carcinoma and extrahepatic metastasis treated with sorafenib. Hepatology research: the official journal of the Japan Society of Hepatology. 2014; 44: $1320-8$.

20. He MK, Le Y, Li QJ, Yu ZS, Li SH, Wei W, et al. Hepatic artery infusion chemotherapy using mFOLFOX versus transarterial chemoembolization for massive unresectable hepatocellular carcinoma: a prospective non-randomized study. Chin J Cancer. 2017; 36: 83.

21. Sergio A, Cristofori C, Cardin R, Pivetta G, Ragazzi R, Baldan A, et al. Transcatheter arterial chemoembolization (TACE) in hepatocellular carcinoma (HCC): the role of angiogenesis and invasiveness. Am J Gastroenterol. 2008; 103: 914-21.

22. Mathonnet M, Descottes B, Valleix D, Labrousse F, Truffinet V, Denizot $Y$. Quantitative analysis using ELISA of vascular endothelial growth factor and basic fibroblast growth factor in human colorectal cancer, liver metastasis of colorectal cancer and hepatocellular carcinoma. World journal of gastroenterology. 2006; 12: 3782-3.

23. Jung $\mathrm{SM}$, Jang JW, You CR, Yoo $\mathrm{SH}$, Kwon JH, Bae $\mathrm{SH}$, et al. Role of intrahepatic tumor control in the prognosis of patients with hepatocellular carcinoma and extrahepatic metastases. Journal of gastroenterology and hepatology. 2012; 27: 684-9.

24. Zhao Y, Wang WJ, Guan S, Li HL, Xu RC, Wu JB, et al. Sorafenib combined with transarterial chemoembolization for the treatment of advanced hepatocellular carcinoma: a large-scale multicenter study of 222 patients. Annals of Oncology. 2013; 24: 1786-92.

25. Cabrera R, Pannu DS, Caridi J, Firpi RJ, Soldevila-Pico C, Morelli G, et al. The combination of sorafenib with transarterial chemoembolisation for hepatocellular carcinoma. Alimentary Pharmacology \& Therapeutics. 2011; 34: 205-13.

26. Xu Y, Shen $\mathrm{Q}$, Wang N, Wu PP, Huang B, Kuang M, et al. Microwave ablation is as effective as radiofrequency ablation for very-early-stage hepatocellular carcinoma. Chin J Cancer. 2017; 36: 14

27. Hiraki T, Yamakado K, Ikeda O, Matsuoka T, Kaminou T, Yamagami T, et al. Percutaneous Radiofrequency Ablation for Pulmonary Metastases from Hepatocellular Carcinoma: Results of a Multicenter Study in Japan. Journal of Vascular and Interventional Radiology. 2011; 22: 741-8.

28. Doreille A, N'Kontchou G, Halimi A, Bouhafs F, Coderc E, Sellier N, et al. Percutaneous treatment of extrahepatic recurrence of hepatocellular carcinoma. Diagnostic and Interventional Imaging. 2016; 97: 1117-23.

29. Li C, Zhang F, Zhang W, Zhang L, Huang Z, Wu P. Feasibility of I-125 brachytherapy combined with sorafenib treatment in patients with multiple 
lung metastases after liver transplantation for hepatocellular carcinoma. Journal of cancer research and clinical oncology. 2010; 136: 1633-40.

30. Liang Z, Wei-jun FAN, Li-gang W, Tian T. Efficacy of $\sim(125) \mathrm{I}$ seed implantation as an adjunctive therapy for extrahepatic metastasis of primary hepatocellular carcinoma. Chinese Journal of Radiology. 2009; 43: 844-8.

31. Yoon YS, Kim HK, Kim J, Choi YS, Shim YM, Paik SW, et al. Long-Term Survival and Prognostic Factors after Pulmonary Metastasectomy in Hepatocellular Carcinoma. Annals of surgical oncology. 2010; 17: 2795-801.

32. Pasqualetti F, Montrone S, Vivaldi C, Zani M, Fedele D, Fornaro L, et al. Stereotactic Body Radiotherapy in Patients with Lung Oligometastases from Colorectal Cancer. Anticancer research. 2017; 37: 315-9. 